\title{
Anharmonicity contributions to the vibrational second hyperpolarizability of conjugated oligomers
}

\author{
Benoît Champagne \\ Laboratoire de Chimie Théorique Appliquée, Facultés Universitaires Notre-Dame de la Paix, \\ Rue de Bruxelles, 61, B-5000 Namur, Belgium \\ Josep M. Luis \\ Laboratoire de Chimie Théorique Appliquée, Facultés Universitaires Notre-Dame de la Paix, Rue de \\ Bruxelles, 61, B-5000 Namur, Belgium and Institute of Computational Chemistry and Department \\ of Chemistry, University of Girona, Campus de Montilivi, 17071 Girona, Catalonia, Spain \\ Miquel Duran and Jose Luis Andrés \\ Institute of Computational Chemistry and Department of Chemistry, University of Girona, \\ Campus de Montilivi, 17071 Girona, Catalonia, Spain \\ Bernard Kirtman \\ Department of Chemistry and Biochemistry, University of California, Santa Barbara, California 93106
}

(Received 15 April 1999; accepted 13 October 1999)

\begin{abstract}
Restricted Hartree-Fock 6-31G calculations of electrical and mechanical anharmonicity contributions to the longitudinal vibrational second hyperpolarizability have been carried out for eight homologous series of conjugated oligomers-polyacetylene, polyyne, polydiacetylene, polybutatriene, polycumulene, polysilane, polymethineimine, and polypyrrole. To draw conclusions about the limiting infinite polymer behavior, chains containing up to 12 heavy atoms along the conjugated backbone were considered. In general, the vibrational hyperpolarizabilities are substantial in comparison with their static electronic counterparts for the dc-Kerr and degenerate four-wave mixing processes (as well as for static fields) but not for electric field-induced second harmonic generation or third harmonic generation. Anharmonicity terms due to nuclear relaxation are important for the dc-Kerr effect (and for the static hyperpolarizability) in the $\sigma$-conjugated polymer, polysilane, as well as the nonplanar $\pi$ systems polymethineimine and polypyrrole. Restricting polypyrrole to be planar, as it is in the crystal phase, causes these anharmonic terms to become negligible. When the same restriction is applied to polymethineimine the effect is reduced but remains quantitatively significant due to the first-order contribution. We conclude that anharmonicity associated with nuclear relaxation can be ignored, for semiquantitative purposes, in planar $\pi$-conjugated polymers. The role of zero-point vibrational averaging remains to be evaluated. (C) 2000 American Institute of Physics. [S0021-9606(00)30602-X]
\end{abstract}

\section{INTRODUCTION}

It is now widely recognized that the effect of vibrations on the (hyper)polarizability must be considered in addressing NLO properties. ${ }^{1,2}$ In contrast with small molecules, the vibrational contribution will, typically be as important as its electronic counterpart ${ }^{3,4}$ for large conjugated organic (or inorganic) molecules and oligomers ${ }^{5-14}$ of interest as NLO materials. In fact, the static vibrational and electronic hyperpolarizabilities of such systems are often approximately equal, ${ }^{15,16}$ although there are also important exceptions. Theoretical attempts ${ }^{17-20}$ to rationalize the near equality have turned out to be flawed. ${ }^{21,22}$ Despite the identification of certain structural factors, ${ }^{4,10}$ which account for some of the exceptions ${ }^{12,13,22}$ and for trends in the relative size of vibrational vs electronic hyperpolarizabilities, the situation remains unresolved at this time.

For large NLO molecules, virtually all the calculations which form the basis for understanding the vibrational first and second hyperpolorizabilities $\left(\beta^{\vee}\right.$ and $\left.\gamma^{\vee}\right)$, as well as their relation to the corresponding electronic quantities $\left(\beta^{e}\right.$ and $\gamma^{e}$ ), have been limited to the (double) harmonic level of approximation. However, the treatment of small molecules indicates that electrical and mechanical anharmonicity effects can sometimes ${ }^{23-33}$ be important and that is the subject of the present paper. In particular, we will examine the second hyperpolarizability of eight different homologous series of conjugated oligomers.

Most previous results for anharmonicity have been obtained using the perturbation theory method of Bishop and Kirtman. ${ }^{34-36}$ (It has recently come to light that a very similar treatment was developed earlier by Flytzanis. ${ }^{37}$ ). This technique requires explicit evaluation of the derivatives of the electrical properties, with respect to vibrational normal coordinates, beyond first order. The vibrational force constants must be determined beyond second-order. Because such calculations are computationally demanding it is difficult to apply the perturbation method directly to the molecules of interest here. Fortunately, there is a finite field (FF) approach that turns out to be more feasible for our purposes. It is based, first, on determining the change in equilibrium 
geometry induced by a finite static electric field. By calculating various electronic properties at the original and relaxed geometries for several field amplitudes one can obtain, through a fitting procedure, the so-called nuclear relaxation (NR) vibrational hyperpolarizabilities. The NR hyperpolarizabilities contain the lowest-order anharmonicity term of each type (to be defined later) that appears in the complete perturbation expression. Very recently, a general FF scheme ${ }^{38}$ for determining these contributions at an arbitrary optical frequency has been presented. However, we have adopted a more practical earlier version, due to Bishop, Hasan, and Kirtman (BHK), ${ }^{39}$ which utilizes the "infinite optical frequency" approximation. In this version the electronic properties that must be evaluated are the dipole moment, $\mu^{e}$, as well as the static polarizability $\alpha^{e}(0 ; 0) \equiv \alpha^{e}$ and first hyperpolarizability, $\beta^{e}(0 ; 0,0) \equiv \beta^{e}$. For a complete treatment of vibrational effects ${ }^{38,40}$ the zero-point vibrational average (ZPVA) of the (hyper) polarizabilities would also be required. At the present time, in order to evaluate the ZPVA it is necessary to explicitly determine anharmonicity parameters. We are, therefore, obliged to save that contribution for the future, leaving us with the NR term. The merits of the infinite optical frequency approximation, as applied to that term, have been demonstrated by Bishop and Dalskov ${ }^{28}$ and, subsequently, by Quinet and Champagne. ${ }^{33}$ A successful implementation of the BHK method, including careful treatment of the Eckart conditions, has very recently been published. ${ }^{41}$

As in previous studies we characterize increasingly large oligomers in order to access the properties of long "polymeric" chains. Eight different series were selected to represent a spectrum of simple polymers that have been targeted in the past for their NLO properties. Polyacetylene (PA) is the prototype $\pi$-conjugated polymer with alternating single and double bonds; polyyne (PY) has alternating single and triple bonds; polydiacetylene (PDA), polybutatriene (PBT), and polycumulene (PC) present different combinations of single, double, and triple bonds; polysilane (PSi) is the prototpe $\sigma$-conjugated polymer; polymethineimine (PMI) is an analog of PA with an asymmetric unit cell; and polypyrrole (PPy) belongs to the class of polyaromatic compounds. Since the ground state geometrical structure of the latter two series is not planar, we have also investigated the planar conformers. Although our primary focus is on anharmonic effects, in some of these cases the double harmonic results are also new. This work is organized as follows: Sec. II summarizes the methodological and computational aspects; it is followed by the results and their discussion in Sec. III; and, finally, our conclusions are given in Sec. IV.

\section{METHODOLOGICAL AND COMPUTATIONAL ASPECTS}

The second hyperpolarizability is the third-order response of the dipole moment to an external electric field, which may have a different magnitude and frequency in each Cartesian direction, ${ }^{42}$

$$
\begin{aligned}
\mu_{\zeta}\left(\omega_{\sigma}\right)= & \mu_{\zeta}^{0}+\sum_{\eta} \alpha_{\zeta \eta}\left(-\omega_{\sigma} ; \omega_{1}\right) E_{\eta}\left(\omega_{1}\right) \\
& +\frac{1}{2} K^{(2)} \sum_{\eta \xi} \beta_{\zeta \eta \xi}\left(-\omega_{\sigma} ; \omega_{1}, \omega_{2}\right) E_{\eta}\left(\omega_{1}\right) E_{\xi}\left(\omega_{2}\right) \\
& +\frac{1}{6} K^{(3)} \sum_{\eta \xi \chi} \gamma_{\zeta \eta \xi \chi}\left(-\omega_{\sigma} ; \omega_{1}, \omega_{2}, \omega_{3}\right) \\
& \times E_{\eta}\left(\omega_{1}\right) E_{\xi}\left(\omega_{2}\right) E_{\chi}\left(\omega_{3}\right)+\cdots
\end{aligned}
$$

Here the subscripts $\eta, \xi$, and $\chi$ denote the direction of the field; $\omega_{1}, \omega_{2}$, and $\omega_{3}$ denote the frequency; and $\omega_{\sigma}=\omega_{1}$ $+\omega_{2}+\omega_{3}$. The static, dc-Kerr, electric field-induced second harmonic generation (dc-SHG), third harmonic generation (THG), and degenerate four-wave mixing or intensitydependent refractive index (IDRI) responses are given by $\gamma(0 ; 0,0,0), \gamma(-\omega ; \omega, 0,0), \gamma(-2 \omega ; \omega, \omega, 0), \gamma(-3 \omega ; \omega, \omega, \omega)$, and $\gamma(-\omega ; \omega,-\omega, \omega)$, respectively. Our focus will be on the case where $\zeta, \eta, \xi$, and $\chi$ all refer to the longitudinal direction, which determines the dominant component of the $\gamma$ tensor. From now on, we simply use a subscript $L$ to indicate this component.

Perturbation theory provides general sum-over(vibronic) states (SOS) expressions for the molecular (hyper)polarizabilities. ${ }^{42}$ The vibrational and electronic contributions are usually separated by applying a canonical or clamped nucleus $(\mathrm{CN})$ approximation, ${ }^{43}$ wherein the two different types of motion are treated sequentially rather than simultaneously. Thus, the electronic (hyper)polarizabilities are calculated with the nuclei clamped in their equilibrium position. Then nuclear motions on the ground state electronic potential energy surface are taken into account. This gives rise to the vibrational hyperpolarizability and the ZPVA correction to the electronic hyperpolarizability. Sometimes the ZPVA correction is considered part of the vibrational hyperpolarizability but, in either event, we ignore the ZPVA here as stipulated earlier. The difference between the exact SOS formulas and the $\mathrm{CN}$ approximation has been analyzed ${ }^{43}$ and a numerical study shows that the error is very small for typical NLO molecules. Therefore, in the present investigation, we employ the $\mathrm{CN}$ approximation.

The properties required for the BHK procedure are $\mu_{L}^{e}$, $\alpha_{L}^{e}$, and $\beta_{L}^{e}$ for different static longitudinal fields, as well as the field-free $\gamma_{L}^{e}$. Except for $\gamma_{L}^{e}$ the values were obtained analytically by the coupled-perturbed Hartree-Fock (CPHF) scheme ${ }^{44}$ implemented in the GAUSSIAN94 program. ${ }^{45} \gamma_{L}^{e}$ was determined by numerical differentiation of the fielddependent $\alpha_{L}^{e}$, which is completely equivalent to an analytical CPHF calculation of the same quantity. The numerical differentiation was carried out by the Romberg procedure ${ }^{46}$ using the fields $2^{k} \times E_{0}$ with $k=0-3$ and $E_{0}=8 \times 10^{-4}$ a.u.

The first step in the vibrational hyperpolarizability calculation is to determine the optimized geometry in the presence of a finite static longitudinal field $E_{L}=E$. Particular care must be exercised to satisfy the field-free Eckart conditions $^{47}$ in order to ensure that the molecule does not reorient during the optimization. This was done using the procedure described in Ref. 41. The longitudinal electronic properties $\mu_{L}^{e}, \alpha_{L}^{e}$, and $\beta_{L}^{e}$ are, then, evaluated at the field- 
dependent optimum geometry. If $P_{L}^{e}\left(E, R_{E}\right)$ is the property value obtained in this manner and $P_{L}^{e}\left(0, R_{0}\right)$ is the corresponding zero-field result, then it can be shown ${ }^{39}$ that the difference between the two is given by

$$
\begin{aligned}
& \mu_{L}^{e}\left(E, R_{E}\right)-\mu_{L}^{e}\left(0, R_{0}\right)=a_{1} E+\frac{1}{2} b_{1} E E+\frac{1}{6} g_{1} E E E+\cdots, \\
& \alpha_{L}^{e}\left(E, R_{E}\right)-\alpha_{L}^{e}\left(0, R_{0}\right)=b_{2} E+\frac{1}{2} g_{2} E E+\cdots \\
& \beta_{L}^{e}\left(E, R_{E}\right)-\beta_{L}^{e}\left(0, R_{0}\right)=g_{3} E+\cdots
\end{aligned}
$$

where $g_{1}, g_{2}, g_{3}$ contain the vibrational hyperpolarizabilities,

$$
\begin{aligned}
& g_{1}=\gamma_{L}^{e}(0 ; 0,0,0)+\gamma_{L}^{\mathrm{v}(r)}(0 ; 0,0,0), \\
& g_{2}=\gamma_{L}^{e}(0 ; 0,0,0)+\gamma_{L}^{\mathrm{v}(r)}(-\omega ; \omega, 0,0)_{\omega \rightarrow \infty}, \\
& g_{3}=\gamma_{L}^{e}(0 ; 0,0,0)+\gamma_{L}^{\mathrm{v}(r)}(-2 \omega ; \omega, \omega, 0)_{\omega \rightarrow \infty} .
\end{aligned}
$$

The superscript $(r)$ on the vibrational hyperpolarizability in Eqs. (5)-(7) indicates that just the nuclear relaxation contribution is included (NR is an alternative notation that has been used) and the subscript $\omega \rightarrow \infty$ denotes the infinite optical frequency approximation.

Values for $g_{1}, g_{2}$, and $g_{3}$ were obtained by numerical differentiation. In this case the usual fitting errors are exacerbated by inaccuracies in the field-dependent geometry optimizations. For this reason it was necessary to lower the default threshold on the residual atomic forces to $10^{-6}$ a.u., along with a SCF threshold of $10^{-12}$ a.u. This gives an accuracy of $10^{-5}-10^{-6}$ in the bond lengths. Despite the very tight geometry optimization, and varying the choice of $E_{0}$ in the Romberg fits, the uncertainty in $g_{1}$ was often fairly large (see later). As expected, $g_{2}$ is more certain and $g_{3}$ even more so.

Taking the limit $\omega \rightarrow \infty$ in the perturbation treatment of Bishop and Kirtman ${ }^{34-36}$ one can show ${ }^{38}$ that the $\gamma_{L}^{\mathrm{v}(r)}$ in Eqs. (5) -(7) can be written as

$$
\begin{aligned}
& \gamma_{L}^{\mathrm{v}(r)}(0 ; 0,0,0)=\left[\alpha^{2}\right]_{L ; \omega=0}^{0,0}+[\mu \beta]_{L ; \omega=0}^{0,0}+\left[\mu^{2} \alpha\right]_{L ; \omega=0}^{\mathrm{I}} \\
& +\left[\mu^{4}\right]_{L ; \omega=0}^{\mathrm{II}}, \\
& \gamma_{L}^{\mathrm{v}(r)}(-\omega ; \omega, 0,0)_{\omega \rightarrow \infty}=\frac{1}{3}\left[\alpha^{2}\right]_{L ; \omega=0}^{0,0}+\frac{1}{2}[\mu \beta]_{L ; \omega=0}^{0,0} \\
& +\frac{1}{6}\left[\mu^{2} \alpha\right]_{L ; \omega=0}^{\mathrm{I}}, \\
& \gamma_{L}^{\mathrm{v}(r)}(-2 \omega ; \omega, \omega, 0)_{\omega \rightarrow \infty}=\frac{1}{4}[\mu \beta]_{L ; \omega=0}^{0,0} .
\end{aligned}
$$

Here the quantity in square brackets identifies the type of term (e.g., $[\mu \beta]$ involves products of a normal coordinate derivative of $\mu$ multiplied by a normal coordinate derivative of $\beta$ ) and the superscript I or II is the total order of perturbation theory. That is to say, if $(n, m)$ denotes the order in electrical $(n)$ and mechanical $(m)$ anharmonicity, I denotes the sum $(0,1)+(1,0)$ while $\mathrm{II}=(0,2)+(2,0)+(1,1)$. Equations (8) -(10) have also been derived by the property expansion method of Luis et al. ${ }^{28-30}$ For the static $\gamma^{\mathrm{v}(r)}$ [cf. Eq. (8)] terms through order II are present; for $\gamma^{\mathrm{v}(r)}(-\omega ; \omega, 0,0)$ the highest-order is reduced to I [see Eq. (9)]; while for $\gamma^{\mathrm{v}(r)}(-2 \omega ; \omega, \omega, 0)$ there is a further reduction to zerothorder [cf. Eq. (10)]. From this pattern it is not surprising that $\gamma^{\mathrm{v}(r)}(-3 \omega ; \omega, \omega, \omega)$ vanishes in the infinite frequency limit.
We note that $\left[\mu^{2} \alpha\right]^{\mathrm{I}}$ is the lowest-order nonvanishing term of that type and the same may be said of $\left[\mu^{4}\right]^{\mathrm{II}}$.

In addition to the terms in Eqs. (8)-(10) the total vibrational hyperpolarizability will contain terms from what is sometimes known as the curvature contribution. These arise from the effect ${ }^{40}$ of NR on the ZPVA corrections to $\mu_{L}^{e}, \alpha_{L}^{e}$, and $\beta_{L}^{e}$ (sometimes the ZPVA itself is included with the other curvature terms). Appropriate formulas may be derived in exactly the same way as Eqs. (5)-(7) are derived from the electronic property expressions, i.e., from Eqs. (2)-(4). The resulting contributions are of exactly the same type as those already present in Eqs. (8) -(10) but two orders of perturbation theory higher. For example, the curvature contribution to $\gamma_{L}^{\mathrm{V}}(-2 \omega ; \omega, \omega, 0)_{\omega \rightarrow \infty}$ is $\frac{1}{4}[\mu \beta]_{L, \omega=0}^{\mathrm{II}}$. As we have already noted neither the curvature contribution nor the ZPVA correction is included in the present treatment.

The vibrational hyperpolarizability $\gamma^{\mathrm{v}(r)}(-\omega ; \omega$, $-\omega, \omega)$ associated with the IDRI may be considered as a special case. From the perturbation treatment ${ }^{34-36}$ it follows that

$\gamma_{L}^{\mathrm{v}(r)}(-\omega ; \omega, \omega,-\omega)_{\omega \rightarrow \infty}=\frac{2}{3}\left[\alpha^{2}\right]_{L ; \omega=0}^{0,0}=2 \sum_{a} \frac{\left(\frac{\partial \alpha_{L}^{e}}{\partial Q_{a}}\right)_{0}^{2}}{\omega_{a}^{2}}$.

In the summation on the far rhs, known as the sum-overmodes (SOM), $Q_{a}$ is a normal coordinate, and $\omega_{a}$ is the corresponding vibrational circular frequency. By combining Eqs. (8)-(10) one can obtain an alternative expression ${ }^{39}$ which is valid through the first-order of perturbation theory. However, we prefer to use the exact relation, i.e., Eq. (11).

The split valence 6-31G basis ${ }^{48}$ has been employed in this study. Although such a basis may be insufficient for small molecules, it becomes more adequate ${ }^{5,49}$ as the size of the quasilinear oligomer is increased because deficiencies due to the limited number of functions on any one atom are counterbalanced by functions located on neighboring atoms. Thus, we can anticipate that for the longer oligomers the 6-31G results will be in good agreement (within a few percent) with those obtained using extended basis sets containing diffuse and polarized functions. Although the errors for the small oligomers are expected to be larger the long chain length behavior, which is our interest, can be adequately determined using the same basis for the entire homologous series.

Of more serious consequence is the omission of electron correlation. For several of the oligomeric series considered here it has been found ${ }^{50}$ that the ratio of the correlated to the Hartree-Fock electronic hyperpolarizability can be large, although this ratio converges much more rapidly with chain length than either the numerator or denominator itself. We expect that the relative importance of the various vibrational hyperpolarizability terms, with respect to each other and/or the static electronic hyperpolarizability, will exhibit a similar behavior as far as the correlated vs Hartree-Fock value is concerned. Thus, our purpose here is twofold. One is to semiquantitatively characterize anharmonicity contributions to the vibrational second hyperpolarizabilities in a represen- 


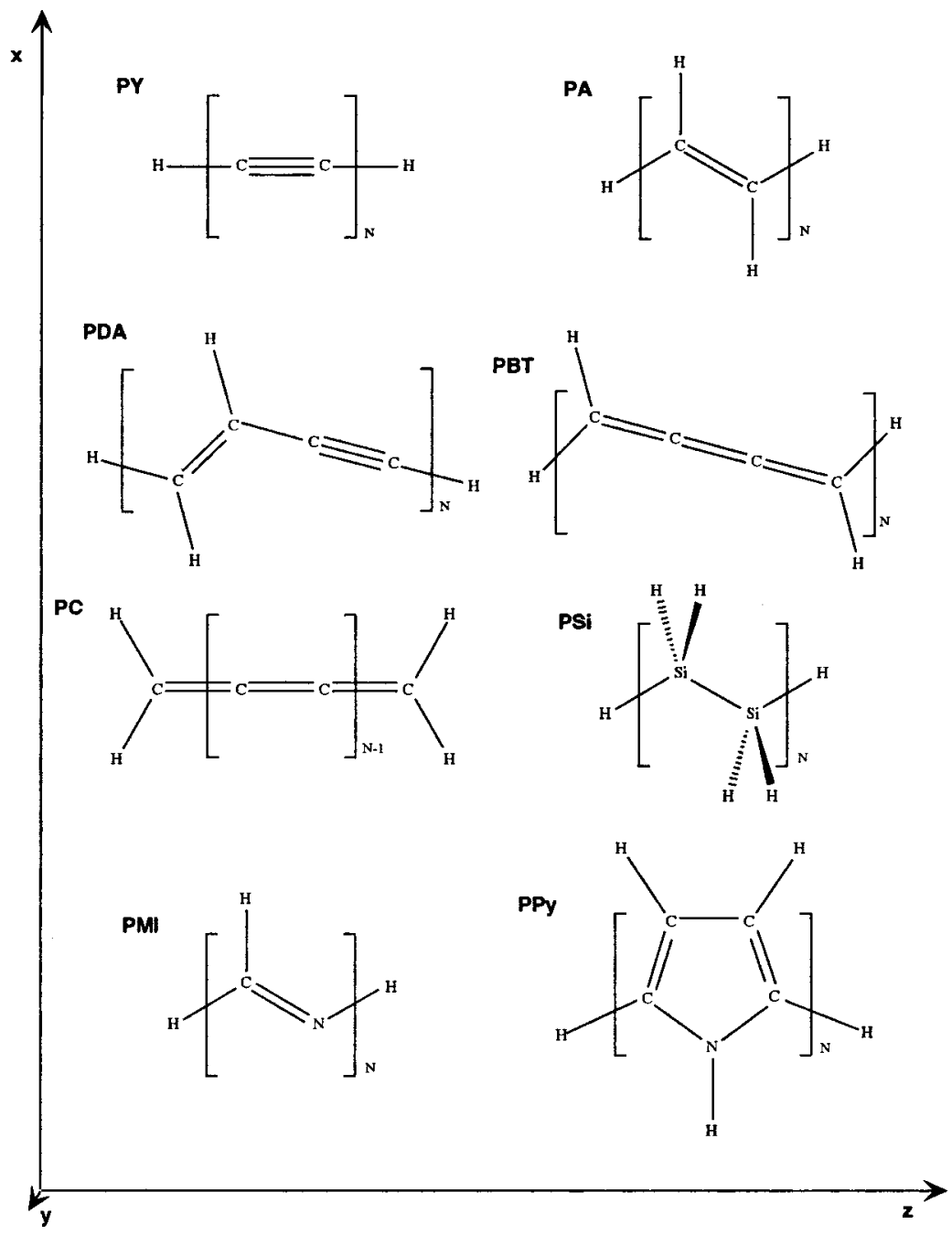

FIG. 1. Representation of the oligomers in Cartesian space.

tative set of conjugated polymers. The other is to establish a baseline for subsequent correlated studies if and when a more quantitative evaluation is desired. At the present time, such studies are prohibitively expensive in terms of our available computational resources.

\section{RESULTS AND DISCUSSION}

The eight homologous series of oligomers that we have examined are displayed in Fig. 1, where $z$ is the longitudinal axis. Since PY and PC are linear the choice of the $z$ direction is obvious. For PA, PDA, PBT, and PSi the longitudinal axis is determined by the line connecting the midpoint of the central bond in the monomer (enclosed in square brackets) with the midpoint of the corresponding bond at the end of the chain. Finally, we employ the longitudinal principal inertial axis for PMI and PPy, which have either a trans-cisoid-like conformation modified by a glide plane operation (PMI) or are helical (PPy). An additional set of calculations was carried out with PMI and PPy restricted to the planar (transcisoid or all-trans) configuration.

In Tables I-VIII we summarize our results for the oligomers shown in Fig. 1. We have only listed those values for $N_{A} \geqslant 8$, where $N_{A}$ is the number of heavy atoms (C, N, Si) along the conjugated backbone; the results for smaller oligomers are available upon request to the authors. The $\gamma_{L}^{\mathrm{v}(r)}$ of
Eqs. (5)-(7) were determined by the FF method. In order to obtain the breakdown into square bracket quantities, the SOM expression given by Eq. (11) was utilized for $\left[\alpha^{2}\right]_{L: \omega=0}^{0,0}$ and, then, Eqs. (8)-(10) were solved for $[\mu \beta]_{L ; \omega=0}^{0,0},\left[\mu^{2} \alpha\right]_{L ; \omega=0}^{\mathrm{I}}$, and $\left[\mu^{4}\right]_{L ; \omega=0}^{\mathrm{II}}$. The values in parentheses give the ratio of the particular quantity (multiplied by 100$)$ with respect to $\gamma_{L}^{e}(0 ; 0,0,0)$. A separation into electrical and mechanical anharmonicity contributions was not made since this would require explicit determination of anharmonicity parameters, which we have assiduously sought to avoid.

In passing we note that $\mathrm{PC}$ has the largest value of $\left|\gamma_{L}^{e}(0 ; 0,0,0)\right|$ if one compares the longest chain in each series. This result is not terribly surprising in view of Morley's ${ }^{51}$ semiempirical results. However, it is premature to conclude that it will remain true for longer oligomers since the value for PBT is also large and grows very rapidly with chain length as shown elsewhere. ${ }^{9}$

Except for PBT and, perhaps, PMI the data of Tables I-VIII indicate that semiquantitative conclusions can be drawn about the behavior of the limiting infinite chain ratio from the results through $N_{A}=12$. Even for PBT the $\gamma^{\mathrm{v}(r)} / \gamma^{e}$ values reported here are more nearly converged than one might be willing to surmise from the numbers in Table IV. We know this on the basis of SOM calculations ${ }^{9}$ that have 
TABLE I. Electronic vs vibrational contributions to the RHF/6-31G longitudinal second hyperpolarizability of polyyne chains of increasing size. The quantity in parentheses is the ratio $\times 100$ with respect to $\gamma_{L}^{e}(0 ; 0,0,0)$. All the values are given in a.u. $\left(1.0\right.$ a.u. of second hyperpolarizability $=6.235377 \times 10^{-65}$ $\mathrm{C}^{4} \mathrm{~m}^{4} \mathrm{~J}^{-3}=7.0423 \times 10^{-54} \mathrm{~m}^{5} \mathrm{~V}^{-2}=5.0367 \times 10^{-40}$ (esu).

\begin{tabular}{lccrc}
\hline \hline & $\gamma_{L}^{e}(0 ; 0,0,0)$ & $\gamma_{L}^{\mathrm{v}(r)}(0 ; 0,0,0)$ & $\gamma_{L}^{\mathrm{v}(r)}(-\omega ; \omega, 0,0)_{\omega \rightarrow \infty}$ & $\gamma_{L}^{\mathrm{v}(r)}(-2 \omega ; \omega, \omega, 0)_{\omega \rightarrow \infty}$ \\
\hline $\mathrm{H}-(\mathrm{C} \equiv \mathrm{C})_{4}-\mathrm{H}$ & 145511 & $1455 \times 10^{2}(100)$ & $486 \times 10^{2}(33)$ & $221(0.2)$ \\
$\mathrm{H}-(\mathrm{C} \equiv \mathrm{C})_{5}-\mathrm{H}$ & 372802 & $3644 \times 10^{2}(98)$ & $1209 \times 10^{2}(32)$ & $598(0.2)$ \\
$\mathrm{H}-(\mathrm{C} \equiv \mathrm{C})_{6}-\mathrm{H}$ & 771259 & $739 \times 10^{3}(96)$ & $2469 \times 10^{2}(32)$ & $1195(0.2)$ \\
& {$\left[\alpha^{2}\right]_{L ; \omega=0}^{0.0}$} & {$[\mu \beta]_{L ; \omega=0}^{0.0}$} & {$\left[\mu^{2} \alpha\right]_{L ; \omega=0}^{\mathrm{I}}$} & {$\left[\mu^{4}\right]_{L ; \omega=0}^{\mathrm{II}}$} \\
\hline $\mathrm{H}-(\mathrm{C} \equiv \mathrm{C})_{4}-\mathrm{H}$ & $143122(98)$ & $882(0.6)$ & $29 \times 10^{2}(2.0)$ & $-14 \times 10^{2}(-1.0)$ \\
$\mathrm{H}-(\mathrm{C} \equiv \mathrm{C})_{5}-\mathrm{H}$ & $357463(96)$ & $2391(0.6)$ & $33 \times 10^{2}(0.9)$ & $12 \times 10^{2}(0.3)$ \\
$\mathrm{H}-(\mathrm{C} \equiv \mathrm{C})_{6}-\mathrm{H}$ & $731420(95)$ & $478 \times 10(0.6)$ & $4 \times 10^{3}(0.5)$ & $-1 \times 10^{3}(-0.2)$ \\
\hline \hline
\end{tabular}

TABLE II. The same as Table I for polyacetylene chains.

\begin{tabular}{ccccc}
\hline \hline & $\gamma_{L}^{e}(0 ; 0,0,0)$ & $\gamma_{L}^{\mathrm{v}(r)}(0 ; 0,0,0)$ & $\gamma_{L}^{\mathrm{v}(r)}(-\omega ; \omega, 0,0)_{\omega \rightarrow \infty}$ & $\gamma_{L}^{\mathrm{v}(r)}(-2 \omega ; \omega, \omega, 0) \omega \rightarrow \infty$ \\
\hline $\mathrm{H}-(\mathrm{CH}=\mathrm{CH})_{4}-\mathrm{H}$ & $21745 \times 10$ & $338 \times 10^{3}(155)$ & $106 \times 10^{3}(49)$ & $-9 \times 10^{2}(-0.4)$ \\
$\mathrm{H}-(\mathrm{CH}=\mathrm{CH})_{5}-\mathrm{H}$ & $60035 \times 10$ & $87 \times 10^{4}(144)$ & $280 \times 10^{3}(47)$ & $-8 \times 10^{2}(-0.1)$ \\
$\mathrm{H}-(\mathrm{CH}=\mathrm{CH})_{6}-\mathrm{H}$ & $131682 \times 10$ & $19 \times 10^{5}(141)$ & $605 \times 10^{3}(46)$ & $18 \times 10^{2}(0.1)$ \\
& {$\left[\alpha^{2}\right]_{L ; \omega=0}^{0.0}$} & {$[\mu \beta]_{L ; \omega=0}^{0.0}$} & {$\left[\mu^{2} \alpha\right]_{L ; \omega=0}^{\mathrm{I}}$} & {$\left[\mu^{4}\right]_{L ; \omega=0}^{\mathrm{II}}$} \\
\hline $\mathrm{H}-(\mathrm{CH}=\mathrm{CH})_{4}-\mathrm{H}$ & $31524 \times 10(145)$ & $-35 \times 10^{2}(-1.7)$ & $16 \times 10^{3}(7)$ & $1 \times 10^{4}(5)$ \\
$\mathrm{H}-(\mathrm{CH}=\mathrm{CH})_{5}-\mathrm{H}$ & $83007 \times 10(138)$ & $-32 \times 10^{2}(-0.5)$ & $3 \times 10^{4}(5)$ & $1 \times 10^{4}(2)$ \\
$\mathrm{H}-(\mathrm{CH}=\mathrm{CH})_{6}-\mathrm{H}$ & $177948 \times 10(135)$ & $72 \times 10^{2}(0.6)$ & $5 \times 10^{4}(4)$ & $2 \times 10^{4}(1)$ \\
\hline \hline
\end{tabular}

TABLE III. The same as Table I for polydiacetylene chains.

\begin{tabular}{ccccr}
\hline \hline & $\gamma_{L}^{e}(0 ; 0,0,0)$ & $\gamma_{L}^{\mathrm{v}(r)}(0 ; 0,0,0)$ & $\gamma_{L}^{\mathrm{v}(r)}(-\omega ; \omega, 0,0)_{\omega \rightarrow \infty}$ & $\left.\gamma_{L}^{\mathrm{v}(r)}(-2 \omega ; \omega, \omega, 0)\right)_{\omega \rightarrow \infty}$ \\
\hline $\mathrm{H}-(\mathrm{CH}=\mathrm{CH}-\mathrm{C} \equiv \mathrm{C})_{2}-\mathrm{H}$ & 160526 & $1759 \times 10^{2}(110)$ & $592 \times 10^{2}(37)$ & $266(0.2)$ \\
$\mathrm{H}-(\mathrm{CH}=\mathrm{CH}-\mathrm{C} \equiv \mathrm{C})_{3}-\mathrm{H}$ & 846831 & $71 \times 10^{4}(84)$ & $284 \times 10^{3}(34)$ & $4175(0.5)$ \\
& {$\left[\alpha^{2}\right]_{L ; \omega=0}^{0.0}$} & {$[\mu \beta]_{L ; \omega=0}^{0.0}$} & {$\left[\mu^{2} \alpha\right]_{L ; \omega=0}^{\mathrm{I}}$} & {$\left[\mu^{4}\right]_{L ; \omega=0}^{\mathrm{II}}$} \\
\hline $\mathrm{H}-(\mathrm{CH}=\mathrm{CH}-\mathrm{C} \equiv \mathrm{C})_{2}-\mathrm{H}$ & $177327(110)$ & $1062(0.7)$ & $-2 \times 10^{3}(-1)$ & $-1 \times 10^{2}(-0.1)$ \\
$\mathrm{H}-(\mathrm{CH}=\mathrm{CH}-\mathrm{C} \equiv \mathrm{C})_{3}-\mathrm{H}$ & $802853(95)$ & $1670 \times 10(2.0)$ & $5 \times 10^{4}(6)$ & $-16 \times 10^{4}(-19)$ \\
\hline \hline
\end{tabular}

TABLE IV. The same as Table I for polybutatriene chains.

\begin{tabular}{ccccc}
\hline \hline & $\gamma_{L}^{e}(0 ; 0,0,0)$ & $\gamma_{L}^{\mathrm{v}(r)}(0 ; 0,0,0)$ & $\gamma_{L}^{\mathrm{v}(r)}(-\omega ; \omega, 0,0)_{\omega \rightarrow \infty}$ & $\gamma_{L}^{\mathrm{v}(r)}(-2 \omega ; \omega, \omega, 0)_{\omega \rightarrow \infty}$ \\
\hline $\mathrm{H}-(\mathrm{CH}=\mathrm{C}=\mathrm{C}=\mathrm{CH})_{2}-\mathrm{H}$ & 83996 & $5813 \times 10^{2}(692)$ & $18230 \times 10(217)$ & $-7191(-8.6)$ \\
$\mathrm{H}-(\mathrm{CH}=\mathrm{C}=\mathrm{C}=\mathrm{CH})_{3}-\mathrm{H}$ & $114839 \times 10$ & $4762 \times 10^{3}(415)$ & $14888 \times 10^{2}(130)$ & $-5090 \times 10(-4.4)$ \\
& {$\left[\alpha^{2}\right]_{L ; \omega=0}^{0.0}$} & {$[\mu \beta]_{L ; \omega=0}^{0.0}$} & {$\left[\mu^{2} \alpha\right]_{L ; \omega=0}^{\mathrm{I}}$} & {$\left[\mu^{4}\right]_{L ; \omega=0}^{\mathrm{II}}$} \\
\hline $\mathrm{H}-(\mathrm{CH}=\mathrm{C}=\mathrm{C}=\mathrm{CH})_{2}-\mathrm{H}$ & $571526(680)$ & $-2876 \times 10(-34)$ & $370 \times 10^{2}(44)$ & $15 \times 10^{2}(1.8)$ \\
$\mathrm{H}-(\mathrm{CH}=\mathrm{C}=\mathrm{C}=\mathrm{CH})_{3}-\mathrm{H}$ & $4608413(401)$ & $-2036 \times 10^{2}(-18)$ & $327 \times 10^{3}(28)$ & $30 \times 10^{3}(2.6)$ \\
\hline \hline
\end{tabular}


TABLE V. The same as Table I for polycumulene chains.

\begin{tabular}{lccrr}
\hline \hline & $\gamma_{L}^{e}(0 ; 0,0,0)$ & $\gamma_{L}^{\mathrm{v}(r)}(0 ; 0,0,0)$ & $\gamma_{L}^{\mathrm{v}(r)}(-\omega ; \omega, 0,0)_{\omega \rightarrow \infty}$ & $\gamma_{L}^{\mathrm{v}(r)}(-2 \omega ; \omega, \omega, 0) \omega \rightarrow \infty$ \\
\hline $\mathrm{H}_{2}-(\mathrm{C}=\mathrm{C})_{4}-\mathrm{H}_{2}$ & -257473 & $27251 \times 10(-106)$ & $880 \times 10^{2}(-34)$ & $-396 \times 10(1.5)$ \\
$\mathrm{H}_{2}-(\mathrm{C}=\mathrm{C})_{5}-\mathrm{H}_{2}$ & -883758 & $696 \times 10^{3}(-79)$ & $2246 \times 10^{2}(-25)$ & $-1129 \times 10(1.3)$ \\
$\mathrm{H}_{2}-(\mathrm{C}=\mathrm{C})_{6}-\mathrm{H}_{2}$ & -2635043 & $155 \times 10^{4}(-59)$ & $499 \times 10^{3}(-19)$ & $-2856 \times 10(1.1)$ \\
& {$\left[\alpha^{2}\right]_{L ; \omega=0}^{0.0}$} & {$[\mu \beta]_{L ; \omega=0}^{0.0}$} & {$\left[\mu^{2} \alpha\right]_{L ; \omega=0}^{\mathrm{I}}$} & {$\left[\mu^{4}\right]_{L ; \omega=0}^{\mathrm{II}}$} \\
\hline $\mathrm{H}_{2}-(\mathrm{C}=\mathrm{C})_{4}-\mathrm{H}_{2}$ & $290399(-113)$ & $-1583 \times 10(6.1)$ & $-5 \times 10^{3}(1.9)$ & $3 \times 10^{3}(-1.2)$ \\
$\mathrm{H}_{2}-(\mathrm{C}=\mathrm{C})_{5}-\mathrm{H}_{2}$ & $747728(-85)$ & $-4515 \times 10(5.1)$ & $-12 \times 10^{3}(1.4)$ & $6 \times 10^{3}(-0.7)$ \\
$\mathrm{H}_{2}-(\mathrm{C}=\mathrm{C})_{6}-\mathrm{H}_{2}$ & $1682887(-64)$ & $-1142 \times 10^{2}(4.3)$ & $-3 \times 10^{4}(1.1)$ & $1 \times 10^{4}(-0.4)$ \\
\hline \hline
\end{tabular}

TABLE VI. The same as Table I for polysilane chains.

\begin{tabular}{lccrr}
\hline \hline & $\gamma_{L}^{e}(0 ; 0,0,0)$ & $\gamma_{L}^{\mathrm{v}(r)}(0 ; 0,0,0)^{\mathrm{a}}$ & $\gamma_{L}^{\mathrm{v}(r)}(-\omega ; \omega, 0,0)_{\omega \rightarrow \infty}^{\mathrm{a}}$ & $\gamma_{L}^{\mathrm{v}(r)}(-2 \omega ; \omega, \omega, 0)_{\omega \rightarrow \infty}$ \\
\hline $\mathrm{H}-\left(\mathrm{SiH}_{2}-\mathrm{SiH}_{2}\right)_{4}-\mathrm{H}$ & 335709 & $108 \times 10^{4}(321)$ & $137 \times 10^{3}(41)$ & $-6010 \times 10(-18)$ \\
$\mathrm{H}-\left(\mathrm{SiH}_{2}-\mathrm{SiH}_{2}\right)_{5}-\mathrm{H}$ & 620245 & $16 \times 10^{5}(258)$ & $204 \times 10^{3}(33)$ & $-947 \times 10^{2}(-15)$ \\
$\mathrm{H}-\left(\mathrm{SiH}_{2}-\mathrm{SiH}_{2}\right)_{6}-\mathrm{H}$ & 967513 & $22 \times 10^{5}(226)$ & $279 \times 10^{3}(29)$ & $-1313 \times 10^{2}(-14)$ \\
& {$\left[\alpha^{2}\right]_{L ; \omega=0}^{0,0}$} & {$[\mu \beta]_{L ; \omega=0}^{0,0}$} & {$\left[\mu^{2} \alpha\right]_{L ; \omega=0}^{\mathrm{I}} \mathrm{a}^{\mathrm{a}}$} & {$\left[\mu^{4}\right]_{L ; \omega=0}^{\mathrm{II}}$} \\
\hline $\mathrm{H}-\left(\mathrm{SiH}_{2}-\mathrm{SiH}_{2}\right)_{4}-\mathrm{H}$ & $443940(132)$ & $-24039 \times 10(-72)$ & $655 \times 10^{3}(195)$ & $22 \times 10^{4}(66)$ \\
$\mathrm{H}-\left(\mathrm{SiH}_{2}-\mathrm{SiH}_{2}\right)_{5}-\mathrm{H}$ & $680742(110)$ & $-3799 \times 10^{2}(-61)$ & $1000 \times 10^{3}(161)$ & $3 \times 10^{5}(48)$ \\
$\mathrm{H}-\left(\mathrm{SiH}_{2}-\mathrm{SiH}_{2}\right)_{6}-\mathrm{H}$ & $940616(97)$ & $-5292 \times 10^{2}(-55)$ & $138 \times 10^{4}(142)$ & $4 \times 10^{5}(41)$ \\
\hline \hline
\end{tabular}

${ }^{\mathrm{a}} \mathrm{See}$ Ref. 53.

TABLE VII. The same as Table I for polypyrrole chains.

\begin{tabular}{ccccc}
\hline \hline & $\gamma_{L}^{e}(0 ; 0,0,0)$ & $\gamma_{L}^{\mathrm{v}(r)}(0 ; 0,0,0)$ & $\gamma_{L}^{\mathrm{v}(r)}(-\omega ; \omega, 0,0)_{\omega \rightarrow \infty}$ & $\gamma_{L}^{\mathrm{v}(r)}(-2 \omega ; \omega, \omega, 0)_{\omega \rightarrow \infty}$ \\
\hline $\mathrm{H}-\left(\mathrm{C}_{4} \mathrm{H}_{3} \mathrm{~N}\right)_{2}-\mathrm{H}$ & 42631 & $692 \times 10^{2}(162)$ & $227 \times 10^{2}(53)$ & $-454(-1.1)$ \\
$\mathrm{H}-\left(\mathrm{C}_{4} \mathrm{H}_{3} \mathrm{~N}\right)_{3}-\mathrm{H}$ & $17788 \times 10$ & $289 \times 10^{3}(163)$ & $1048 \times 10^{2}(59)$ & $-23 \times 10(-0.1)$ \\
& {$\left[\alpha^{2}\right]_{L ; \omega=0}^{0,0}$} & {$[\mu \beta]_{L ; \omega=0}^{0,0}$} & {$\left[\mu^{2} \alpha\right]_{L ; \omega=0}^{\mathrm{I}}$} & {$\left[\mu^{4}\right]_{L ; \omega=0}^{\mathrm{II}}$} \\
\hline $\mathrm{H}-\left(\mathrm{C}_{4} \mathrm{H}_{3} \mathrm{~N}\right)_{2}-\mathrm{H}$ & $73193(172)$ & $-1817(-4.3)$ & $-45 \times 10^{2}(-11)$ & $24 \times 10^{2}(5.6)$ \\
$\mathrm{H}-\left(\mathrm{C}_{4} \mathrm{H}_{3} \mathrm{~N}\right)_{3}-\mathrm{H}$ & $35461 \times 10(199)$ & $-91 \times 10(-0.5)$ & $-777 \times 10^{2}(-44)$ & $13 \times 10^{3}(7.5)$ \\
\hline \hline
\end{tabular}

TABLE VIII. The same as Table I for polymethineimine chains.

\begin{tabular}{ccccc}
\hline \hline & $\gamma_{L}^{e}(0 ; 0,0,0)$ & $\gamma_{L}^{\mathrm{v}(r)}(0 ; 0,0,0)$ & $\gamma_{L}^{\mathrm{v}(r)}(-\omega ; \omega, 0,0)_{\omega \rightarrow \infty}$ & $\gamma_{L}^{\mathrm{v}(r)}(-2 \omega ; \omega, \omega, 0)_{\omega \rightarrow \infty}$ \\
\hline $\mathrm{H}-(\mathrm{CH}=\mathrm{N})_{4}-\mathrm{H}$ & 48315 & $92 \times 10^{4}(19 \times 100)$ & $843 \times 10^{2}(174)$ & $270 \times 10(5.6)$ \\
$\mathrm{H}-(\mathrm{CH}=\mathrm{N})_{5}-\mathrm{H}$ & 102715 & $20 \times 10^{5}(19 \times 100)$ & $175 \times 10^{3}(170)$ & $50 \times 10^{2}(4.9)$ \\
$\mathrm{H}-(\mathrm{CH}=\mathrm{N})_{6}-\mathrm{H}$ & 182714 & $36 \times 10^{5}(20 \times 100)$ & $33 \times 10^{4}(18 \times 10)$ & $107 \times 10^{2}(5.9)$ \\
& {$\left[\alpha^{2}\right]_{L ; \omega=0}^{0,0}$} & {$[\mu \beta]_{L ; \omega=0}^{0,0}$} & {$\left[\mu^{2} \alpha\right]_{L ; \omega=0}^{\mathrm{I}}$} & {$\left[\mu^{4}\right]_{L ; \omega=0}^{\mathrm{II}}$} \\
\hline $\mathrm{H}-(\mathrm{CH}=\mathrm{N})_{4}-\mathrm{H}$ & $80476(167)$ & $108 \times 10^{2}(22.4)$ & $312 \times 10^{3}(646)$ & $51 \times 10^{4}(11 \times 100)$ \\
$\mathrm{H}-(\mathrm{CH}=\mathrm{N})_{5}-\mathrm{H}$ & $168016(164)$ & $201 \times 10^{2}(19.6)$ & $651 \times 10^{3}(634)$ & $11 \times 10^{5}(11 \times 100)$ \\
$\mathrm{H}-(\mathrm{CH}=\mathrm{N})_{6}-\mathrm{H}$ & $324272(177)$ & $428 \times 10^{2}(23.4)$ & $12 \times 10^{5}(65 \times 10)$ & $21 \times 10^{5}(11 \times 100)$ \\
\hline \hline
\end{tabular}


TABLE IX. The same as Table I for all-planar polypyrrole chains.

\begin{tabular}{lcccc}
\hline \hline & $\gamma_{L}^{e}(0 ; 0,0,0)$ & $\gamma_{L}^{\mathrm{v}(r)}(0 ; 0,0,0)$ & $\gamma_{L}^{\mathrm{v}(r)}(-\omega ; \omega, 0,0)_{\omega \rightarrow \infty}$ & $\gamma_{L}^{\mathrm{v}(r)}(-2 \omega ; \omega, \omega, 0)_{\omega \rightarrow \infty}$ \\
\hline $\mathrm{H}-\left(\mathrm{C}_{4} \mathrm{H}_{3} \mathrm{~N}\right)_{2}-\mathrm{H}$ & 50057 & $43 \times 10^{3}(86)$ & $140 \times 10^{2}(28)$ & $-156(-0.3)$ \\
$\mathrm{H}-\left(\mathrm{C}_{4} \mathrm{H}_{3} \mathrm{~N}\right)_{3}-\mathrm{H}$ & $23436 \times 10$ & $244 \times 10^{3}(104)$ & $75 \times 10^{3}(32)$ & $89 \times 10(0.4)$ \\
& {$\left[\alpha^{2}\right]_{L ; \omega=0}^{0,0}$} & {$[\mu \beta]_{L ; \omega=0}^{0,0}$} & {$\left[\mu^{2} \alpha\right]_{L ; \omega=0}^{\mathrm{I}}$} & {$\left[\mu^{4}\right]_{L ; \omega=0}^{\mathrm{II}}$} \\
\hline $\mathrm{H}-\left(\mathrm{C}_{4} \mathrm{H}_{3} \mathrm{~N}\right)_{2}-\mathrm{H}$ & $40377(81)$ & $-622(-1.2)$ & $54 \times 10^{2}(11)$ & $-2 \times 10^{3}(-4.3)$ \\
$\mathrm{H}-\left(\mathrm{C}_{4} \mathrm{H}_{3} \mathrm{~N}\right)_{3}-\mathrm{H}$ & $209380(89)$ & $356 \times 10(1.5)$ & $20 \times 10^{3}(9.5)$ & $11 \times 10^{3}(5.3)$ \\
\hline \hline
\end{tabular}

been done for the IDRI and dc-SHG properties, which have only double harmonic contributions. In the case of IDRI, chain lengths up to 28 carbon atoms were previously ${ }^{9}$ considered. They show a slow decrease of $2\left[\alpha^{2}\right]_{L ; \omega=0}^{0,0} /$ $3 \gamma_{L}^{e}(0 ; 0,0,0)$ from 2.67 at $N_{A}=12$ to 2.24 at 28 carbons. In the case of dc-SHG a value for the corresponding $\gamma^{\mathrm{v}(r)} / \gamma^{e}$ ratio $(\times 100)$ of -3.2 was obtained for 16 carbons compared to -4.4 at $N_{A}=12$ (cf. Table IV). Thus, in the infinite polymer limit, $\gamma^{\mathrm{v}(r)}$ will be substantially more important than the static electronic property as far as the IDRI process is concerned, whereas exactly the opposite is true for dc-SHG. Assuming similar convergence behavior for $\gamma_{L}^{\mathrm{v}(r)}(0 ; 0,0,0)$ and $\gamma_{L}^{\mathrm{v}(r)}(-\omega ; \omega, 0,0)_{\omega \rightarrow \infty}$, then the former will be considerably larger than $\gamma_{L}^{e}(0 ; 0,0,0)$ in the infinite polymer limit of PBT while the latter will be comparable in size. For PMI the $\gamma^{\mathrm{v}(r)} / \gamma^{l}$ vs $N_{A}$ curves wiggle slightly in the region $N_{A}$ $=8-12$. This is due to numerical roundoff errors and/or conformational effects rather than a harbinger of unusual long chain behavior. $^{52}$

Before moving on to discuss anharmonicities a few observations are in order regarding the two $\gamma^{\mathrm{v}(r)}$ that are completely determined at the doubly harmonic level of approximation, namely, the IDRI and dc-SHG. For the longest oligomer in each series the magnitude of the $\gamma^{\mathrm{v}(r)}$ contribution to the IDRI varies between $43 \%$ and $267 \%$ of the static electronic term and, therefore, should always be taken into account. The largest value occurs for PBT which has the smallest average BLA (Ref. 9) along the backbone with the exception of PC. It is known that the IDRI of PBT is due primarily to intense Raman-active $k=0$ modes which create substantial variations of the bond length alternation pattern along the conjugated backbone. ${ }^{6,8,9,11}$ Other oligomers where the ratio is over $100 \%$ are PMI and PPy; their vibrational hyperpolarizabilities have not been considered previously. It will be shown further on that, for PMI, the large value is due to torsional motions.

In contrast with the IDRI, the magnitude of $\gamma_{L}^{\mathrm{v}(r)}(-2 \omega ; \omega, \omega, 0)_{\omega \rightarrow \infty}$ is always less than $14 \%$ (PSi). This means that for most purposes the latter is negligible compared to the electronic term. In PSi $\gamma_{L}^{\mathrm{v}(r)}(-2 \omega ; \omega, \omega, 0)_{\omega \rightarrow \infty}$ has been attributed, ${ }^{14}$ for the most part, to $\mathrm{H}$-wagging modes which induce substantial electron density polarization along the chain.

From Eqs. (10) and (11) $\gamma_{L}^{\mathrm{v}(r)}(-2 \omega ; \omega, \omega, 0)_{\omega \rightarrow \infty} /$ $\gamma_{L}^{\mathrm{v}(r)}(-\omega ; \omega,-\omega, \omega)_{\omega \rightarrow \infty}=3[\mu \beta]_{L ; \omega=0}^{0,0} / 8\left[\alpha^{2}\right]_{L ; \omega=0}^{0,0}$. For the longest chain in each oligomer series, other than PSi, the maximum value of $[\mu \beta]_{L ; \omega=0}^{0,0} /\left[\alpha^{2}\right]_{L ; \omega=0}^{0,0}$ is 0.14 , i.e., the maximum value of $\gamma_{L}^{\mathrm{v}(r)}(-2 \omega ; \omega, \omega, 0)_{\omega \rightarrow \infty} /$
$\gamma_{L}^{\mathrm{v}(r)}(-\omega ; \omega,-\omega, \omega)_{\omega \rightarrow \infty}$ is about 0.05 . If one writes $\gamma_{L}^{\mathrm{v}(r)}(-\omega ; \omega, 0,0)_{\omega \rightarrow \infty}$ in the form [cf. Eq. (9)],

$\gamma_{L}^{\mathrm{v}(r)}(-\omega ; \omega, 0,0)_{\omega \rightarrow \infty}$

$$
=\frac{1}{3}\left[\alpha^{2}\right]_{L ; \omega=0}^{0,0}\left[1+\frac{3}{2} \frac{[\mu \beta]_{L ; \omega=0}^{0,0}}{\left[\alpha^{2}\right]_{L ; \omega=0}^{0,0}}+\frac{1}{2} \frac{\left[\mu^{2} \alpha\right]_{L ; \omega=0}^{1}}{\left[\alpha^{2}\right]_{L ; \omega=0}^{0,0}}\right],
$$

then it is clear that the $[\mu \beta]_{L ; \omega=0}^{0,0}$ term will make a relatively small contribution to the dc-Kerr $\gamma^{\mathrm{v}(r)}$ (i.e., $<20 \%$ ) except, again, for PSi. In the case of PSi there is a high degree of cancellation between the first two terms on the rhs of Eq. (12) which causes the anharmonic term to predominate. This is consistent with results found earlier by Perpète et al. ${ }^{14,53}$

As we have just seen, anharmonicity plays a crucial role in the vibrational dc-Kerr effect of PSi due to the nearcancellation of two large zeroth-order terms. This situation does not occur in the $\pi$-conjugated oligomers. Nonetheless, the role of anharmonicity in the dc-Kerr effect could still be very important. The fractional contribution of the $\left[\mu^{2} \alpha\right]_{L ; \omega=0}^{I}$ term to $\gamma_{L}^{\mathrm{v}(r)}(-\omega ; \omega, 0,0)_{\omega \rightarrow \infty}$ is given by $\left[\mu^{2} \alpha\right]_{L ; \omega=0}^{I} / 6 \gamma_{L}^{\mathrm{v}(r)}(-\omega ; \omega, 0,0)_{\omega>\infty}$. For the longest oligomer $\left(N_{A}=12\right)$ in each series the magnitude of this ratio is less than 0.075 except for PSi (0.87), PMI (0.60), and PPy (0.12). Although the PPy value for $N_{A}=12$ is not very large, it is still increasing rapidly with chain length at that point and, therefore, we cannot assume it will be inconsequential for longer oligomers. One question of interest is whether the relatively large values for PMI and PPy arise as a result of torsional motions. These motions, and the torsional equilibrium configuration as well, may be strongly affected by solid state packing forces. In order to determine the answer to the above question we undertook a set of calculations with the oligomers restricted to $\sigma_{h}$ symmetry. The $\sigma_{h}$ symmetry restriction preserves planarity and, thereby removes out-ofplane torsions. Our results are reported in Tables IX and X. If the PMI oligomers are forced to be in the trans-cisoid planar conformation, the ratio $\left[\mu^{2} \alpha\right]_{L ; \omega=0}^{I} / 6 \gamma_{L}^{\mathrm{v}(r)}$ $(-\omega ; \omega, 0,0)_{\omega \rightarrow \infty}$ for the longest oligomer decreases from 0.60 to 0.39 , which is still substantial. For all-trans PPy the corresponding ratio is reduced to less than 0.05 and, therefore, the contribution from modes other than torsion is negligible.

Finally, we examine the contribution of anharmonicity to $\gamma_{L}^{\mathrm{v}(r)}(0 ; 0,0,0)$. In this case there are both first- and secondorder terms. The relative importance of anharmonicity is de- 
TABLE X. The same as Table I for all-planar polymethineimine chains.

\begin{tabular}{lcccc}
\hline \hline & $\gamma_{L}^{e}(0 ; 0,0,0)$ & $\gamma_{L}^{\mathrm{v}(r)}(0 ; 0,0,0)$ & $\gamma_{L}^{\mathrm{v}(r)}(-\omega ; \omega, 0,0)_{\omega \rightarrow \infty}$ & $\gamma_{L}^{\mathrm{v}(r)}(-2 \omega ; \omega, \omega, 0)_{\omega \rightarrow \infty}$ \\
\hline $\mathrm{H}-(\mathrm{CH}=\mathrm{N})_{4}-\mathrm{H}$ & 81806 & $164 \times 10^{3}(200)$ & $350 \times 10^{2}(43)$ & $-1042 \times 10(-13)$ \\
$\mathrm{H}-(\mathrm{CH}=\mathrm{N})_{5}-\mathrm{H}$ & 208383 & $470 \times 10^{3}(226)$ & $100 \times 10^{3}(48)$ & $-2242 \times 10(-11)$ \\
$\mathrm{H}-(\mathrm{CH}=\mathrm{N})_{6}-\mathrm{H}$ & 424922 & $110 \times 10^{4}(253)$ & $234 \times 10^{3}(55)$ & $-3820 \times 10(-9)$ \\
& {$\left[\alpha^{2}\right]_{L ; \omega=0}^{0,0}$} & {$[\mu \beta]_{L ; \omega=0}^{0,0}$} & {$\left[\mu^{2} \alpha\right]_{L ; \omega=0}^{\mathrm{I}}$} & {$\left[\mu^{4}\right]_{L ; \omega=0}^{\mathrm{II}}$} \\
\hline $\mathrm{H}-(\mathrm{CH}=\mathrm{N})_{4}-\mathrm{H}$ & $134127(164)$ & $-4169 \times 10(-51)$ & $668 \times 10^{2}(82)$ & $5 \times 10^{3}(6)$ \\
$\mathrm{H}-(\mathrm{CH}=\mathrm{N})_{5}-\mathrm{H}$ & $327796(157)$ & $-8969 \times 10(-43)$ & $215 \times 10^{3}(103)$ & $2 \times 10^{4}(9)$ \\
$\mathrm{H}-(\mathrm{CH}=\mathrm{N})_{6}-\mathrm{H}$ & $660794(155)$ & $-15320 \times 10(-36)$ & $543 \times 10^{3}(128)$ & $3 \times 10^{4}(7)$ \\
\hline \hline
\end{tabular}

termined by the magnitude of the ratio $\left\{\left[\mu^{2} \alpha\right]_{L ; \omega=0}^{\mathrm{I}}\right.$ $\left.+\left[\mu^{4}\right]_{L ; \omega=0}^{\mathrm{II}}\right\} / \gamma_{L}^{\mathrm{V}(r)}(0 ; 0,0,0)$. Again it is convenient to look at the largest oligomer in each series. Then we see that the anharmonicity effect is relatively small (magnitude of ratio $<0.16)$ except for PSi (0.80), PMI (0.92), and PPy (-0.22). For PPy and PSi this is due to the first-order term but for PMI the second-order term is $40 \%$ larger. Once more the $\sigma_{h}$ symmetry-restricted calculations shed light on the role of torsional motions in PMI and PPY. In both oligomers the magnitude of the ratio is reduced by almost 50\%. For PMI this is due primarily to the fact that the second order term becomes quite small. In the case of PPy the first-order term remains more important than the second-order term but changes sign.

\section{CONCLUSIONS}

We have carried out an exploratory RHF/6-31G investigation of anharmonicity contributions to the longitudinal vibrational second hyperpolarizability in eight homologous series of conjugated oligomers. Chains containing up to 12 heavy atoms along the conjugated backbone were considered. This proved sufficient for us to draw qualitative or semiquantitative conclusions about the limiting infinite polymer behavior. In general, the vibrational hyperpolarizabilities are substantial in comparison with their electronic counterparts for the static, dc-Kerr, and IDRI processes but not for dc-SHG or THG. Anharmonicity is important for the dcKerr effect and for the static hyperpolarizability in the $\sigma$-conjugated polymer, PSi, as well as the nonplanar $\pi$ systems PMI and PPy. Restricting PPy to a planar configuration, as can be induced by crystal packing forces, causes the anharmonic terms to become negligible. When the same restriction is applied to PMI the effect of anharmonicity is reduced but still quantitatively significant due to the firstorder contribution. We draw the conclusion that anharmonicity effects due to nuclear relaxation can be ignored, at least for semiquantitative purposes, in planar $\pi$-conjugated polymers. On the contrary, these effects need to be taken into account for $\sigma$-conjugated and nonplanar $\pi$-conjugated polymers. The role of ZPVA and of higher order curvature terms remains to be considered.

\section{ACKNOWLEDGMENTS}

The authors thank Professor J. M. André, Dr. E. A. Perpète, Dr. D. Jacquemin, and O. Quinet for discussions. B. C. thanks the Belgian National Fund for Scientific Research for his Research Associate position. J. M. L. thanks the Belgian National Interuniversity Research Program on "Sciences of Interfacial and Mesoscopic Structures"' (PAI/IUAP No. P4/ 10) and the Generalitat de Catalunya through the CIRIT project No. FI/95/5101 for financially supporting his stay in the CTA lab. The calculations have been performed on the IBM SP2 of the Namur Scientific Computing Facility (Namur-SCF) for which the authors gratefully acknowledge the financial support of the FNRS-FRFC, the "Loterie Nationale" for the convention No. 2.4519.97 and the Belgian National Interuniversity Research Program on "Sciences of Interfacial and Mesoscopic Structures" (PAI/IUAP No. $\mathrm{P} 4 / 10)$.

${ }^{1}$ D. M. Bishop, Adv. Chem. Phys. 104, 1 (1998).

${ }^{2} \mathrm{~B}$. Kirtman, in Theoretical and Computational Modeling of $N L O$ and Electronic Materials, American Chemical Society Series, edited by S. P. Karna and A. T. Yeates (American Chemical Society, New York, 1996), Vol. 628, p. 58.

${ }^{3}$ B. Kirtman and B. Champagne, Int. Rev. Phys. Chem. 16, 389 (1997).

${ }^{4}$ B. Champagne and B. Kirtman, Chem. Phys. 245, 213 (1999).

${ }^{5}$ B. Kirtman and M. Hasan, J. Chem. Phys. 96, 470 (1992).

${ }^{6}$ B. Kirtman, B. Champagne, and J. M. André, J. Chem. Phys. 104, 4125 (1996).

${ }^{7}$ B. Champagne, Chem. Phys. Lett. 261, 57 (1996).

${ }^{8}$ B. Champagne, E. A. Perpète, J. M. André, and B. Kirtman, Synth. Met. 85, 1047 (1997).

${ }^{9}$ E. A. Perpète, B. Champagne, and B. Kirtman, J. Chem. Phys. 107, 2463 (1997).

${ }^{10}$ B. Champagne, Int. J. Quantum Chem. 65, 689 (1997).

${ }^{11}$ E. A. Perpète, B. Champagne, J. M. André, and B. Kirtman, J. Mol. Struct. 425, 115 (1998).

${ }^{12}$ B. Champagne, E. A. Perpète, Th. Legrand, D. Jacquemin, and J. M. André, J. Chem. Soc., Faraday Trans. 94, 1547 (1998).

${ }^{13}$ B. Champagne, Th. Legrand, E. A. Perpète, O. Quinet, and J. M. André, Collect. Czech. Chem. Commun. 63, 1295 (1998).

${ }^{14}$ E. A. Perpète, J. M. André, and B. Champagne, J. Chem. Phys. 109, 4624 (1998).

${ }^{15}$ P. Zuliani, M. Del Zoppo, C. Castiglioni, G. Zerbi, S. R. Marder, and J. W. Perry, J. Chem. Phys. 103, 9935 (1995).

${ }^{16}$ P. Zuliani, M. Del Zoppo, C. Castiglioni, G. Zerbi, C. Andraud, T. Brotin, and A. Collet, J. Phys. Chem. 99, 16242 (1995).

${ }^{17}$ C. Castiglioni, M. Del Zoppo, and G. Zerbi, Phys. Rev. B 53, 13319 (1996).

${ }^{18}$ H. S. Kim, M. Cho, and S. J. Jeon, J. Chem. Phys. 107, 1936 (1997).

${ }^{19}$ A. Painelli, Chem. Phys. Lett. 285, 352 (1998).

${ }^{20}$ H. Torii, K. Furuya, and M. Tasumi, J. Phys. Chem. 102, 8422 (1998).

${ }^{21}$ D. M. Bishop and B. Kirtman, Phys. Rev. B 56, 2273 (1997).

${ }^{22}$ D. M. Bishop, B. Champagne, and B. Kirtman, J. Chem. Phys. 109, 9987 (1998).

${ }^{23}$ Martí, J. L. Andrés, J. Bertrán, and M. Duran, Mol. Phys. 80, 625 (1993).

${ }^{24}$ D. M. Bishop, B. Kirtman, H. A. Kurtz, and J. E. Rice, J. Chem. Phys. 98, 8024 (1993).

${ }^{25}$ D. M. Bishop, J. Pipin, and B. Kirtman, J. Chem. Phys. 102, 6778 (1995).

${ }^{26}$ D. M. Bishop and J. Pipin, J. Chem. Phys. 103, 4980 (1995). 
${ }^{27}$ D. M. Bishop and E. K. Dalskov, J. Chem. Phys. 104, 1004 (1996).

${ }^{28}$ J. M. Luis, M. Duran, and J. L. Andrès, J. Chem. Phys. 107, 1501 (1997).

${ }^{29}$ J. M. Luis, J. Martí, M. Duran, and J. L. Andrés, Chem. Phys. 217, 29 (1997).

${ }^{30}$ J. M. Luis, J. Martí, M. Duran, J. L. Andrés, and B. Kirtman, J. Chem. Phys. 108, 4123 (1998).

${ }^{31}$ B. Champagne, Chem. Phys. Lett. 287, 185 (1998).

${ }^{32}$ D. M. Bishop, F. L. Gu, and S. M. Cybulski, J. Chem. Phys. 109, 8407 (1998).

${ }^{33}$ O. Quinet and B. Champagne, J. Chem. Phys. 109, 10594 (1998).

${ }^{34}$ D. M. Bishop and B. Kirtman, J. Chem. Phys. 95, 2646 (1991).

${ }^{35}$ D. M. Bishop and B. Kirtman, J. Chem. Phys. 97, 5255 (1992).

${ }^{36}$ D. M. Bishop, J. M. Luis, and B. Kirtman, J. Chem. Phys. 108, 10013 (1998).

${ }^{37}$ C. Flytzanis, in Quantum Electronics, edited by H. Rabin and C. L. Tang (Academic, New York, 1975), Vol. IA, p. 9.

${ }^{38}$ D. M. Bishop and B. Kirtman, J. Chem. Phys. 109, 9674 (1998).

${ }^{39}$ D. M. Bishop, M. Hasan, and B. Kirtman, J. Chem. Phys. 103, 4157 (1995).

${ }^{40}$ B. Kirtman, J. M. Luis, and D. M. Bishop, J. Chem. Phys. 108, 10008 (1998).

${ }^{41}$ J. M. Luis, M. Duran, J. L. Andrés, B. Champagne, and B. Kirtman, J. Chem. Phys. 111, 875 (1999).

${ }^{42}$ B. J. Orr and J. F. Ward, Mol. Phys. 20, 513 (1971); D. M. Bishop, J. Chem. Phys. 100, 6535 (1994).

${ }^{43}$ D. M. Bishop, B. Kirtman, and B. Champagne, J. Chem. Phys. 107, 5780 (1997).

${ }^{44}$ P. W. Langhoff, M. Karplus, and R. P. Hurst, J. Chem. Phys. 44, 505 (1966); T. C. Caves and M. Karplus, ibid. 50, 3649 (1969); C. E. Dykstra and P. G. Jasien, Chem. Phys. Lett. 109, 388 (1984); H. Sekino and R. J. Bartlett, J. Chem. Phys. 85, 976 (1986); S. P. Karna and M. Dupuis, J. Comput. Chem. 12, 487 (1991).

${ }^{45}$ Gaussian 94, Revision B.1, M. J. Frisch, G. W. Trucks, H. B. Schlegel, P.
M. W. Gill, B. G. Johnson, M. A. Robb, J. R. Cheeseman, T. Keith, G. A. Petersson, J. A. Montgomery, K. Raghavachari, M. A. Al-Laham, V. G. Zakrzewski, J. V. Ortiz, J. B. Foresman, J. Cioslowski, B. B. Stefanov, A. Nanayakkara, M. Challacombe, C. Y. Peng, P. Y. Ayala, W. Chen, M. W. Wong, J. L. Andres, E. S. Replogle, R. Gomperts, R. L. Martin, D. J. Fox, J. S. Binkley, D. J. Defrees, J. Baker, J. P. Stewart, M. Head-Gordon, C. Gonzalez, and J. A. Pople, Gaussian, Inc., Pittsburgh, Pennsylvania, 1995.

${ }^{46}$ P. J. Davis and P. Rabinowitz, Numerical Integration (Blaisdell, London, 1967), p. 166; D. Jacquemin, B. Champagne, and J. M. André, Int. J. Quantum Chem. 65, 679 (1997).

${ }^{47}$ C. Eckart, Phys. Rev. 47, 552 (1935); S. R. Polo, J. Chem. Phys. 24, 1133 (1956).

${ }^{48}$ W. J. Hehre, R. Ditchfield, and J. A. Pople, J. Chem. Phys. 56, 2257 (1972).

${ }^{49}$ G. J. B. Hurst, M. Dupuis, and E. Clementi, J. Chem. Phys. 89, 385 (1988).

${ }^{50}$ J. L. Toto, T. T. Toto, C. P. de Melo, M. Hasan, and B. Kirtman, Chem. Phys. Lett. 244, 59 (1995); J. L. Toto, T. T. Toto, and C. P. de Melo, ibid. 245, 660 (1995); J. L. Toto, T. T. Toto, C. P. de Melo, and K. Robins, J. Chem. Phys. 102, 8048 (1995).

${ }^{51}$ J. O. Morley, J. Phys. Chem. 99, 10166 (1995).

${ }^{52}$ E. A. Perpète and D. Jacquemin (unpublished).

${ }^{53}$ The $\left[\mu^{2} \alpha\right]_{L ; \omega=0}^{\mathrm{I}}$ and $\left[\mu^{4}\right]_{L ; \omega=0}^{\mathrm{II}}$ results for PSi differ from those reported in Ref. 14 because in the latter case, the finite field optimization procedure was carried out by blocking the central $\mathrm{Si}-\mathrm{Si}$ bond so as to align the field-free geometry along the longitudinal axis. Blocking the central bond prevents the Eckart conditions from being satisfied and, therefore, the FF results do not match the SOM values. Nevertheless, when the chain length grows this "blocking" approach becomes suitable because the direction of the field-induced dipole moment coincides with the longitudinal axis and no Eckart rotation is necessary. 